\title{
Management of antiretroviral-related neuropsychiatric adverse effects
}

\author{
Anita Rachlis MD MEd FRCPC
}

$\mathrm{T}$ he introduction of highly active antiretroviral therapy has led to sustained suppression of viral replication, immune reconstitution and improved clinical outcomes. Clinical trials $(1,2)$ have informed recommendations for specific regimens as preferred therapy in treatment-naïve HIV-infected individuals. Observational cohort studies (3-5) have indicated that the rate of viral rebound after initial suppression to an undetectable (less than 50 copies $/ \mathrm{mL}$ ) viral load varies according to specific antiretroviral agents. In these cohort studies, efavirenz in combination with a dual nucleoside backbone continues to be an effective third agent. In addition, clinical trials (6-8) have demonstrated that efavirenz can be combined in a nucleoside-sparing regimen with boosted protease inhibitors, and can be successfully substituted for protease inhibitors and maintain viral suppression (9). Efavirenz is generally well tolerated, with the most frequent side effects being neuropsychiatric, including dizziness, insomnia, somnolence, impaired concentration and vivid dreams. These symptoms are usually mild to moderate, usually self-limiting, and resolve after the first two to four weeks of efavirenz use. In one clinical trial (10), central nervous system (CNS) symptoms occurred in more than $50 \%$ of efavirenz-treated patients. In a substudy of the ACTG 5095 trial (A5095 [11]) in which patients were randomly assigned to receive efavirenz with or without abacavir, $6 \%$ of those patients receiving efavirenz experienced CNS symptoms or mood disorders that led to discontinuation of the agent. Symptoms occurred within the first week of therapy in those on efavirenz; however, these resolved within the first month with no evidence of significant differences in neuropsychological performance. No significant changes in anxiety or depressed mood were found (11-14). Longer term toxicity has been observed with persistence of neuropsychiatric disorders after a mean of two years on efavirenz therapy, but these disturbances were mild and clinically tolerable, and did not impair the patients' quality of life and psychological status (15). Among efavirenz-treated patients, serious psychiatric events have been reported, such as severe depression, suicidal ideation, nonfatal suicide attempts, aggressive behaviour, paranoid reactions and manic reactions, all of which occurred in low frequency (16). Hence, management of depression and related neuropsychiatric symptoms associated with HIV infection and antiretroviral therapy is essential.

In the A5095 study, there was an association with higher efavirenz trough blood levels and neurological symptoms, but this occurred only in the first week of treatment. Another study (17) confirmed these findings: patients with higher efavirenz trough blood levels were at increased risk of CNS side effects. The neuropsychiatric toxicities have been associated with a CYP2B6 (G516T) genotype and other polymorphisms, which affect drug clearance, resulting in higher blood concentration levels. The CYP2B6516 TT genotype may be found in 20\% of African-American patients (18-20). CYP2B6 genotyping can lead to recognition of patients at risk of neuropsychiatric symptoms and to targeted interventions with the initiation of efavirenz therapy. The role of therapeutic drug monitoring to decrease the incidence of these symptoms requires evaluation.

\section{REFERENCES}

1. Panel on Clinical Practices for Treatment of HIV Infection. Guidelines for the Use of Antiretroviral Agents in HIV-1-Infected Adults and Adolescents. US Department of Health and Human Services (DHHS). October 10, 2006. < http://www.aidsinfo.nih.gov/ ContentFiles/AdultandAdolescentGL.pdf $>$ (Version current at October 27, 2006).

2. Hammer SM, Saag MS, Schechter M, et al; International AIDS Society-USA panel. Treatment for adult HIV infection: 2006 recommendations of the International AIDS Society-USA Panel. JAMA 2006;296:827-43.

3. Phillips AN, Ledergerber B, Horban A, et al; EuroSIDA Study Group. Rate of viral rebound according to specific drugs in the regimen in 2120 patients with HIV suppression. AIDS 2004;18:1795-804.

4. Smith CJ, Phillips AN, Hill T, et al; United Kingdom Collaborative HIV Cohort Study Group. The rate of viral rebound after attainment of an HIV load $<50$ copies $/ \mathrm{mL}$ according to specific antiretroviral drugs in use: Results from a multicenter cohort study. J Infect Dis 2005;192:1387-97.

5. Torti C, Maggiolo F, Patroni A, et al; MASTER Cohort. Exploratory analysis for the evaluation of lopinavir/ritonavir-versus efavirenz-based HAART regimens in antiretroviral-naïve HIV-positive patients: Results from the Italian MASTER Cohort. J Antimicrob Chemother 2005;56:190-5.

6. Tebas P, Zhang H, Yarasheski K, et al. Switch to protease inhibitorcontaining/nucleoside reverse transcriptase inhibitor-sparing regimen increases appendicular fat and serum lipid levels without affecting glucose metabolism or bone mineral density. The results of a prospective randomized trial, ACTG 5125S. 12th Conference on Retroviruses and Opportunistic Infections. Boston, February 22 to 25, 2005. (Abst 40)

7. Ferré V, Allavena C, Poizot-Martin I, et al. BIKS study (lopinavir/ritonavir-efavirenz combination): Complete 24-week results. Antivir Ther 2003;8(Suppl 1):S193.

8. Valantin M-A, Lanoy E, Bentata M, et al. Maintenance of virological suppression and impact on lipoatrophy of NRTI-sparing regimen in antiretroviral treated patients: NONUKE ANRS 108 Study. 10th European AIDS Conference. Dublin, November 17 to 20, 2005. (Abst PS5/2)

9. Becker S, Rachlis A, Gill J, et al. Successful substitution of protease inhibitors with Sustiva (efavirenz) in patients with undetectable viral loads - a prospective, randomized, multicenter, open-label study (DMP 049). 8th Conference on Retroviruses and Opportunistic Infections. Chicago, February 4 to 8, 2001. (Abst 20)

10. Staszewski S, Morales-Ramirez J, Tashima KT, et al; Study 006 Team. Efavirenz plus zidovudine and lamivudine, efavirenz plus indinavir, and indinavir plus zidovudine and lamivudine in the treatment of HIV-1 infection in adults. N Engl J Med 1999;341:1865-73.

Division of Infectious Diseases, Department of Medicine, Sunnybrook Health Sciences Centre, University of Toronto, Toronto, Ontario Correspondence: Dr Anita Rachlis, Department of Medicine, Sunnybrook Health Sciences Centre, 2075 Bayview Avenue, Room A226, Toronto, Ontario M4N 3M5. Telephone 416-480-4689, fax 416-480-5808, e-mail a.rachlis@utoronto.ca 
11. Clifford DB, Evans S, Yang Y, et al. Impact of efavirenz on neuropsychological performance and symptoms in HIV-infected individuals. Ann Intern Med 2005;143:714-21.

12. Perez-Molina JA. Safety and tolerance of efavirenz in different antiretroviral regimens: Results from a national multicenter prospective study in 1,033 HIV-infected patients. HIV Clin Trials 2002;3:279-86.

13. Puzantian T. Central nervous system adverse effects with efavirenz: Case report and review. Pharmacotherapy 2002;22:930-3.

14. Clifford D, Evans S, Yang Y, Gulick R; A5097S Team. Long-term neuropsychological effects of efavirenz-based treatment.

13th Conference on Retroviruses and Opportunistic Infections. Denver, February 5 to 8, 2006. (Abst 773)

15. Fumaz CR, Munoz-Moreno JA, Molto J, et al. Long-term neuropsychiatric disorders on efavirenz-based approaches: Quality of life, psychologic issues, and adherence. J Acquir Immune Defic Syndr 2005;38:560-5.
16. Lochet P, Peyriere H, Lotthe A, Mauboussin JM, Delmas B, Reynes J. Long-term assessment of neuropsychiatric adverse reactions associated with efavirenz. HIV Med 2003;4:62-6.

17. Gutierrez F, Navarro A, Padilla S, et al. Prediction of neuropsychiatric adverse events associated with long-term efavirenz therapy, using plasma drug monitoring. Clin Infect Dis 2005;41:1648-53.

18. Haas DW, Ribaudo HJ, Kim RB, et al. Pharmacogenetics of efavirenz and central nervous system side effects: An Adult AIDS Clinical Trials Group study. AIDS 2004;18:2391-400.

19. Ribaudo HJ, Haas DW, Tierney C, et al; Adult AIDS Clinical Trials Group Study. Pharmacogenetics of plasma efavirenz exposure after treatment discontinuation: An Adult AIDS Clinical Trials Group Study. Clin Infect Dis 2006;42:401-7.

20. Nolan D, Phillips E, Mallal S. Efavirenz and CYP2B6 polymorphism: Implications for drug toxicity and resistance. Clin Infect Dis 2006;42:408-10. 


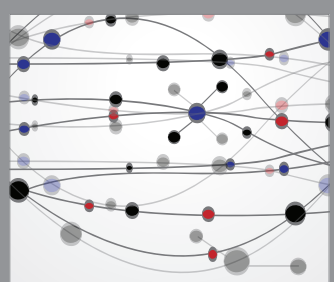

The Scientific World Journal
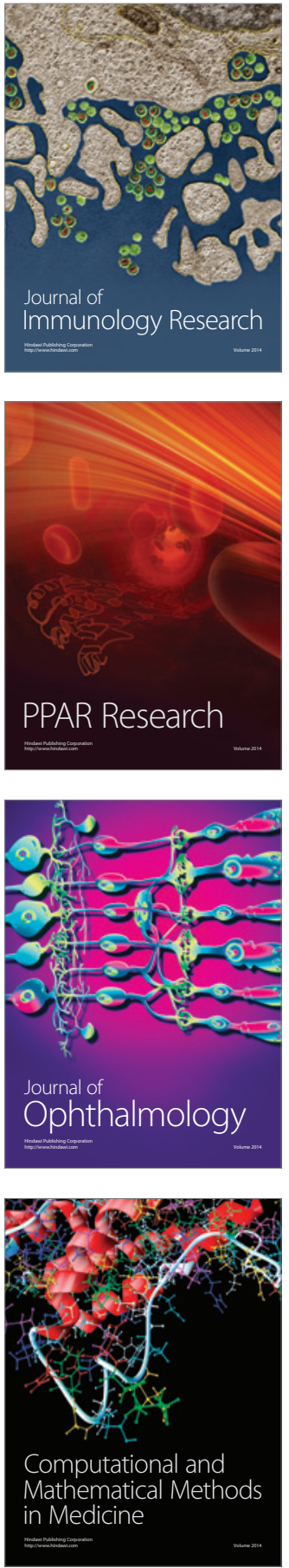

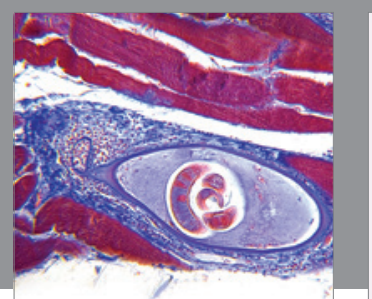

Gastroenterology Research and Practice

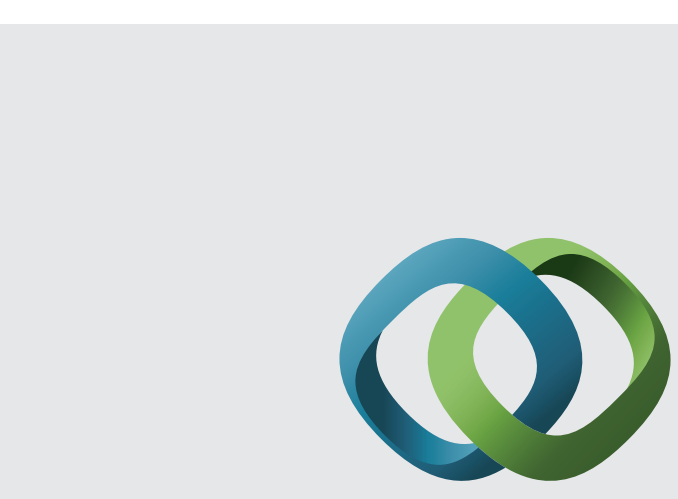

\section{Hindawi}

Submit your manuscripts at

http://www.hindawi.com
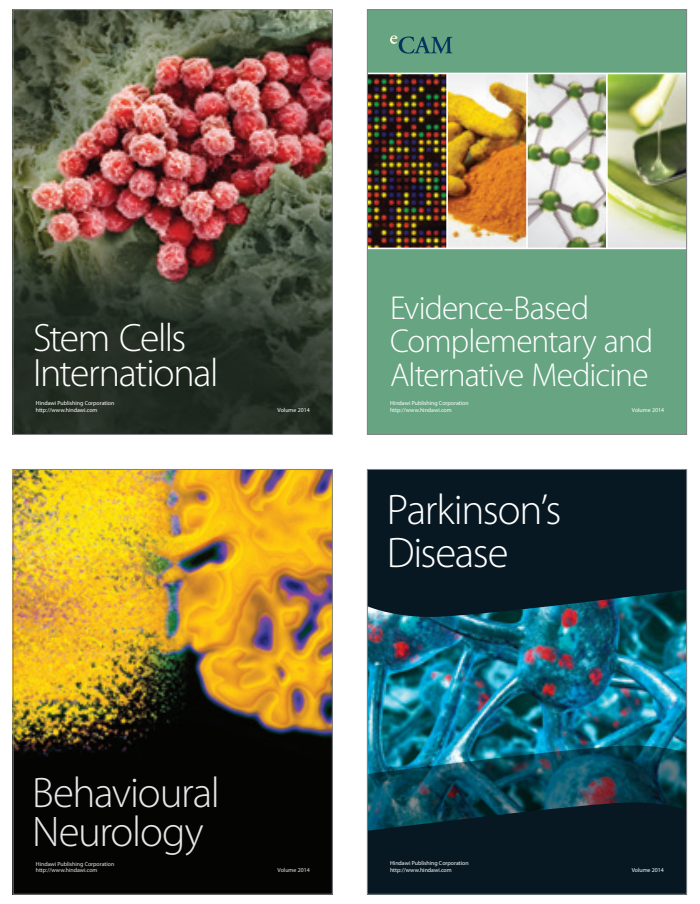
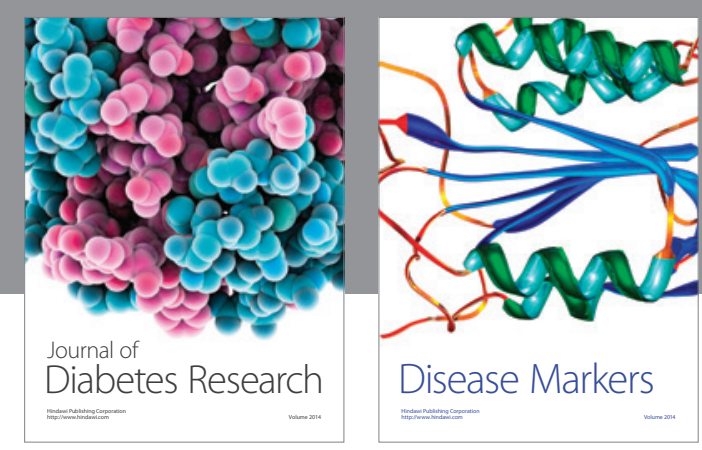

Disease Markers
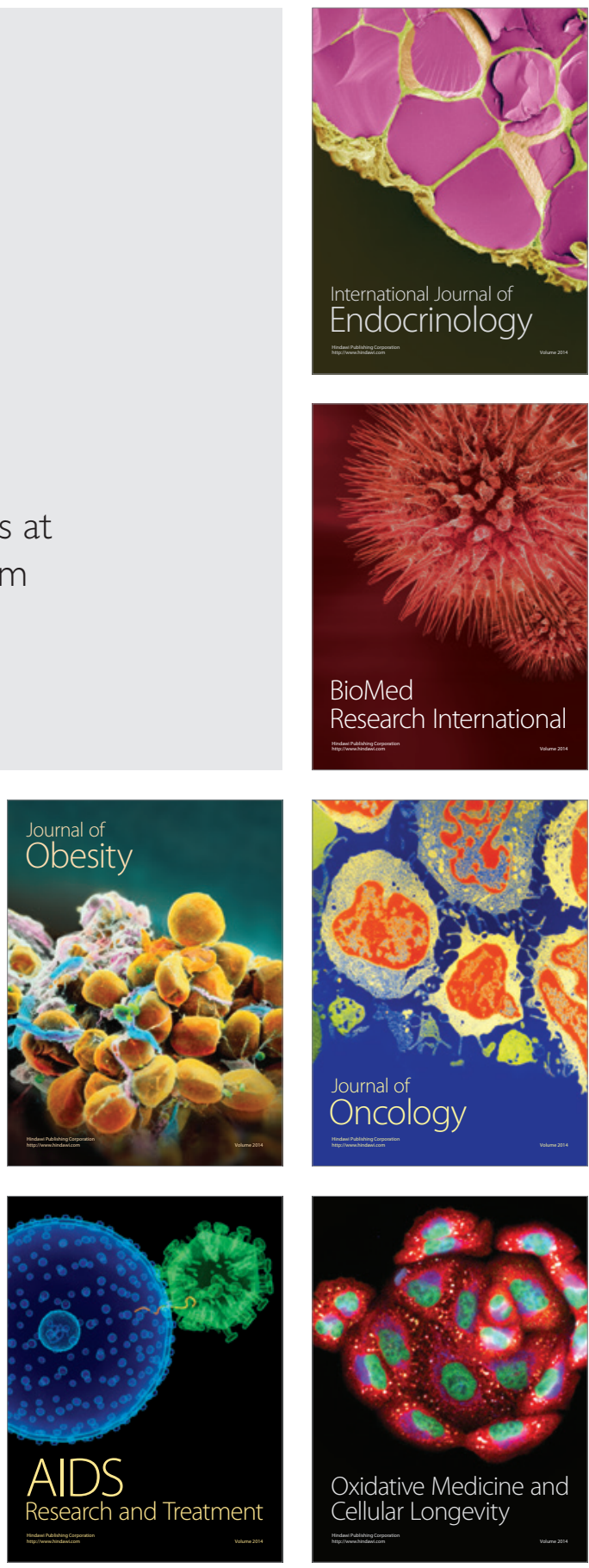